\title{
Analysis of the bolt performance with shear force
}

\author{
Dawei Mao ${ }^{1,2}$, Yu Chen ${ }^{1, *}$ \\ ${ }^{1}$ Hunan Provincial Key Laboratory of Hydropower Development Key Technology, Changsha, Hunan province, 410014, China \\ ${ }^{2}$ PowerChina Zhongnan Engineering Corporation Limited, Changsha, Hunan province, 410014, China
}

\begin{abstract}
The bolt anchorage performance with shear force has been analysed by analytical method in this paper. By coding in Matlab, the loop and the judgment statement can be used to control the displacement angle. The force-displacement curve of the analysis model from the beginning of loading to the failure of the bolt under a certain displacement loading angle is obtained. In the test and field, the values of displacement and force before failure and the load-displacement curves before the peak value can be obtained by using the program with the parameters. The bolt deformation in different strength surrounding rock mass and the anchoring influence of the rock bolt with different diameters have been studied. The resultant displacement increases linearly with the increase of the bolt, and the resultant force increases exponentially with the increase of the bolt.
\end{abstract}

\section{Introduction}

The bolt is not only an important element to transfer the induced tensile stress of rock mass, but also an additional resistance to shear failure. In recent years, more and more attention has been paid to the anchorage performance of bolts with the influence of tensile and shear loads.

Ren [1] studied an analytical method to evaluate the performance of the grouting bolt in the whole process. The analytical solution consists of five stages from elastic to plastic stages. The analytical model proposed by Ma [2] not only considers the decoupling mechanism of bolt materials, but also the elasticity and post-yielding behaviour of bolt materials. He [3] studied the analytical model of bolts with the influence of rock joint aperture increments, which can obtain the elongation of bolts under different tensions. These models depict the mechanism behaviour of the interface between rock and bolt. In addition, it reveals the deformation change of the grouted bolt with tensile stress. The deformation characteristics of the bolt under tensile and shear stress have been modified as well.

When the bolt installed in rock is influenced by the shear deformation, the rock bolt will bend and the rock or grout will react accordingly. The compression load distribution applied by the rock or grout depends on the rock or grout. Couples of simplified distribution forms are proposed [4-6]. Liu [7] investigated an analytical method of the stress distribution along with the bolt through the sliding surface by combining the beam model on elastic foundation and the bolt pull-out model. It analysed the load and deformation behaviour of the deflection section and developed a mechanical model.
Figure 1 displays the bolt support effect of rock in rock slopes [8-11]. Bolt can resist shear failure in weak plane and the sliding in rock mass. The deformation direction of the rock mass is not completely consistent with the bolt, which results in the normal and tangential forces in the bolt body.

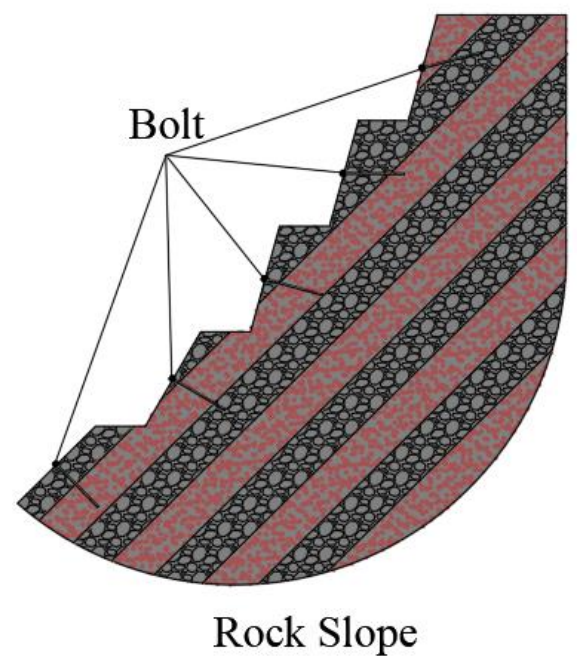

Fig. 1. Illustration of bolt support in rock slope.

In practical condition, especially in the deep rock mass, joints are often open and the cracks may appear between composite strata supported by bolts. Most of the models mentioned above are concentrated on closed joints, and the effect of sliding friction resistance cannot be ignored in shear strength. Therefore, there is considerable space to establish a unified analytical model of reinforced bolts with tension and shear. In this paper, an analytical method of anchoring rock mass under tensile and shear forces is proposed and performed

\footnotetext{
$\overline{\text { * Corresponding author: joyidol@163.com }}$
} 
in Matlab program. Therefore, the ultimate force ultimate displacement curves obtained from the analytical and tests have been verified. The parameters of rock and bolt are also discussed.

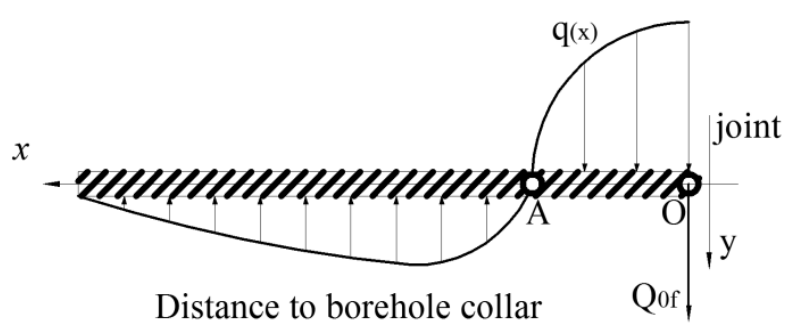

Fig. 2. Illustration of elastic stress of rock bolt with surrounding rock mass.

As shown in Figure 2, point $\mathrm{O}$ is the point of anchor in the node, point $\mathrm{A}$ is the neutral point, and $\mathrm{Q}(\mathrm{x})$ is the force acting on the side of bolt with surrounding rock. According to the research of Pellet and Egger [4], the force acting on the rock mass in contact with the bolt is directly proportional to the deformation deflection of the bolt. According to Newton's third theorem, the lateral force of bolt and the force acting on the contact surface of surrounding rock are equal to the larger reverse interaction force. Therefore, the deformation of the bolt conforms to the Winkler foundation beam formula.

According to lots of pull-out tests and the changing law of the shear stress between the bolt and the grout from the outside to the inside, the full-length bonded bolt performance can be divided into four stages: complete decoupling, partial decoupling, partial decoupling, and residual resistance. The shear strength increases linearly to the peak strength without decoupling. This paper discusses the shear deformation of the bolt caused by the mutual displacement of the rock mass on both sides of the joint. In fact, these two movements are mutually perpendicular. On the one hand, it can be seen that the two movements are independent of each other. On the other hand, the change of the axial shear model caused by the compression and separation of the bolt and the grout should also be considered.

\section{Analytical method of the bolt with shear force}

According to the theory, the bolt failure may occur at the maximum bending moment point (A) or the maximum resultant force point $(\mathrm{O})$. In the test and field, the bolt failure generally occurs at the point $\mathrm{O}$. Based on the Tresca yield criterion, the relationship is shown in Equation 1. The tension force $\left(\mathrm{N}_{\mathrm{p}}\right)$ and shear force $\left(\mathrm{Q}_{\mathrm{p}}\right)$ can be coupled in an elliptic curve.

$$
\left(\mathrm{N}_{0}\right)^{2}+\left(2 \mathrm{Q}_{0}\right)^{2}=\left(\mathrm{A} \sigma_{\mathrm{S}}\right)^{2}
$$

As shown in Figure 3, the bolt performance with the pull force and shear force can be analysed. The angle between the axial direction of the bolt and the bolt tangent at point $\mathrm{O}$ is defined as $\theta$. At point $\mathrm{O}, \mathrm{F}_{0 \mathrm{f}}$ is the final force and $\mathrm{D}_{\text {of }}$ is the final deformation. In addition, Equations (2-4) indicate the directions of pull force and shear force, where $\mathrm{L}_{\mathrm{i}}$ is the elongation caused by bending, $\mathrm{L}_{t}$ is the elongation caused by joint, $\mathrm{L}_{\mathrm{r}}$ is the elongation caused by bolt rotation, $\delta_{\mathrm{L}}$ is the ultimate elongation of the bolt, $\mathrm{y}(0)$ is the deflection at point $\mathrm{O}$.

$$
\begin{aligned}
& \mathrm{D}_{p}=L_{t} \cos \theta \\
& \mathrm{D}_{s}=y(0)+L_{t} \sin \theta \\
& \delta_{L}=L_{i}+L_{t}+L_{r}
\end{aligned}
$$

Through compiling $M$ file technique in Matlab, the previous formula can be written into it. We may get the pull force and displacement by given the shear force $\mathrm{Q}_{0}$. If continually increasing the shear force $\mathrm{Q}_{0}$ until tension and shear forces satisfy Equations (5-6).

$$
\begin{aligned}
& \mathrm{F}_{t o t}=\sqrt{N_{0}^{2}+Q_{0}^{2}} \\
& \mathrm{D}_{\text {tot }}=\sqrt{D_{0 p}^{2}+D_{0 s}^{2}}
\end{aligned}
$$

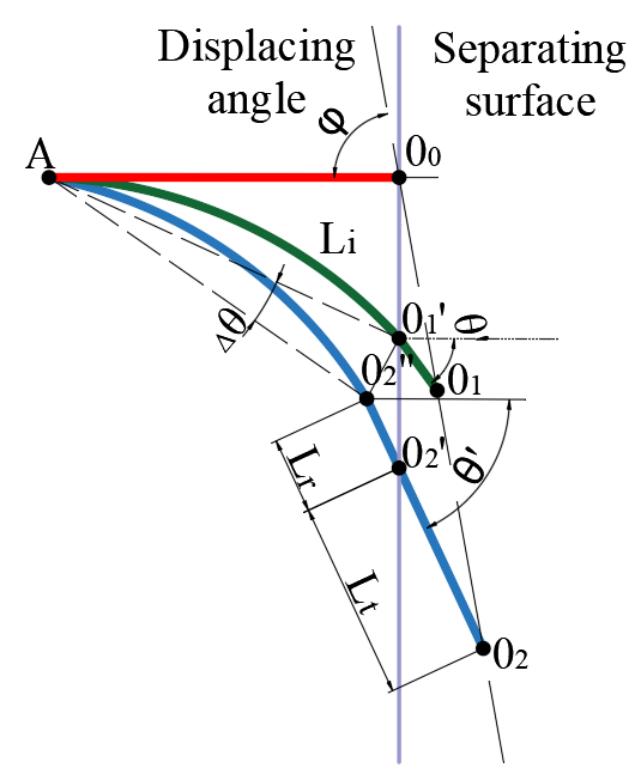

Fig. 3. The description of bolt failure.

\section{Verification of the analytical and test results}

The calculated results are planned to compare with the experiment results [12]. The concrete test block is a 1-mlong block with strength of $30 \mathrm{MPa}$. A number of boreholes with a diameter of about $40 \mathrm{~mm}$ are distributed in the middle, which are connected by grouting with a 20 mm diameter threaded steel bolt.

According to the material characteristics, the parameters in the formula are set as follows: the radius of the anchor rod is $20 \mathrm{~mm}$; the total length is $1 \mathrm{~m}$; the elastic modulus $\mathrm{E}$ is $100 \mathrm{GPa} ; \mathrm{E}_{1}$ is one tenth of $\mathrm{E}$; the tensile strength is $650 \mathrm{MPa}$; the yield strength is $600 \mathrm{MPa}$; the length and width of concrete block are $1 \mathrm{~m}$; the compressive strength is $30 \mathrm{MPa}$; the elastic modulus is $4 \mathrm{GPa}$; the Poisson's ratio is 0.25 . The grouting parameters are the same as those of concrete block, bolt and grouting. The friction coefficient between slurries is 
0.5 by considering the influence of thread. The calculated results under the same displacement angle are summarized as follows: A, B, C and D are the results under the displacement angles of $0^{\circ}, 20^{\circ}, 40^{\circ}$ and $60^{\circ}$ respectively.

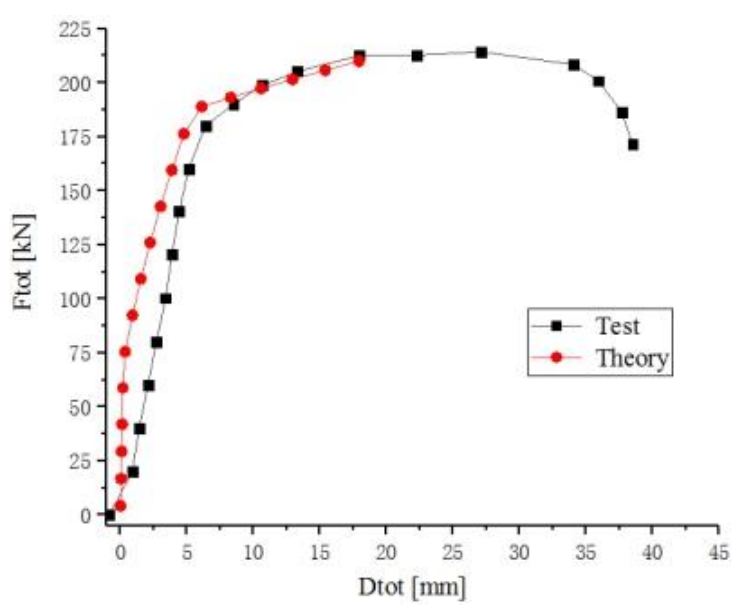

a. $0^{\circ}$ loading angle

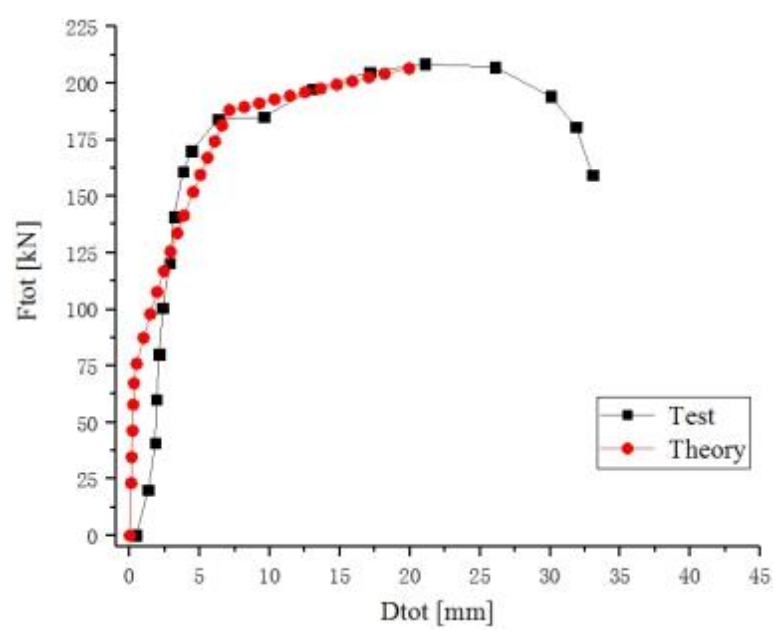

b. $40^{\circ}$ loading angle

Fig. 4. Comparison between analytical and test results.

According to the comparison of $0^{\circ}$ and $40^{\circ}$ with the curves obtained by the formula and experiments in Figure 4, it can judge whether the bolt is damaged or not. In addition, the curve fitting has a high degree of consistency, which confirms the accuracy of the formula. In the test and field, the values of displacement and force before failure and the load-displacement curves before the peak value can be obtained by using this small program.

After fitting with a certain state of the test, the different parameters can be compared according to the parametric study. It is to predict the deformation of the rock bolt in different strength surrounding rock and the anchoring influence of the bolt with different diameters. Figure 5 shows the relationship between displacement (a) and resultant force (b) when the bolt is in failure under $60^{\circ}$ loading angle. Obviously, the resultant displacement and resultant force decrease slowly with the increase of rock strength and tend to a stable value. Figure 6 shows the sum displacement (a diagram) and resultant force (b) when the bolt diameter is changed under $45^{\circ}$ loading angle. It is obvious that the resultant displacement increases linearly with the increase of the bolt, and the resultant force increases exponentially with the increase of the bolt.

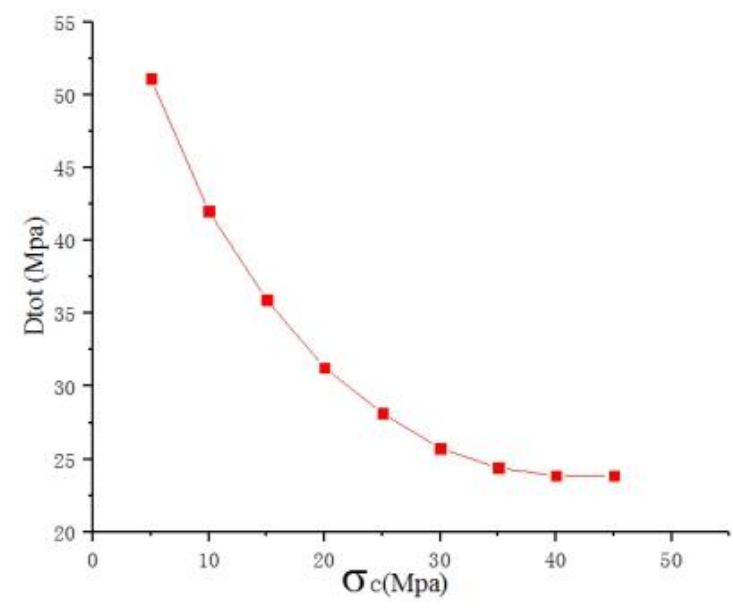

a. Resultant displacement under different rock strength

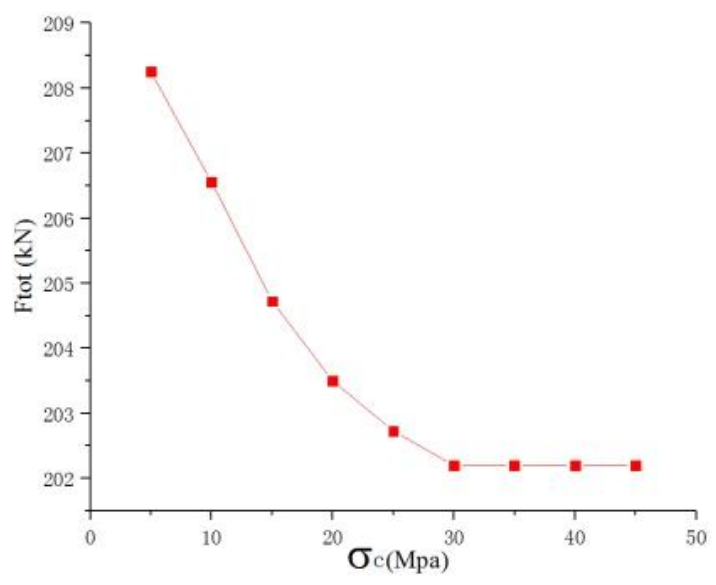

b. Resultant force under different rock strength

Fig. 5. Variation of resultant force and resultant displacement of different rock strength under $60^{\circ}$ loading angle.

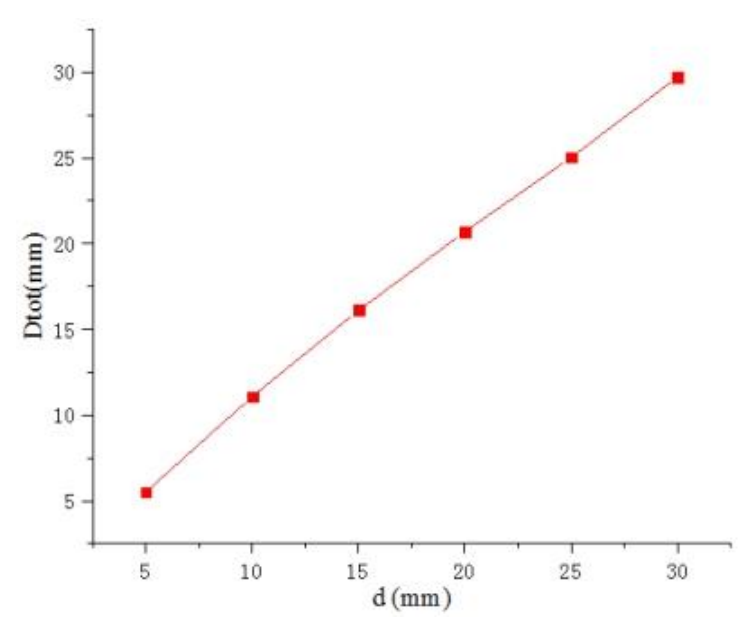

a. Combined displacement of different bolt diameter 


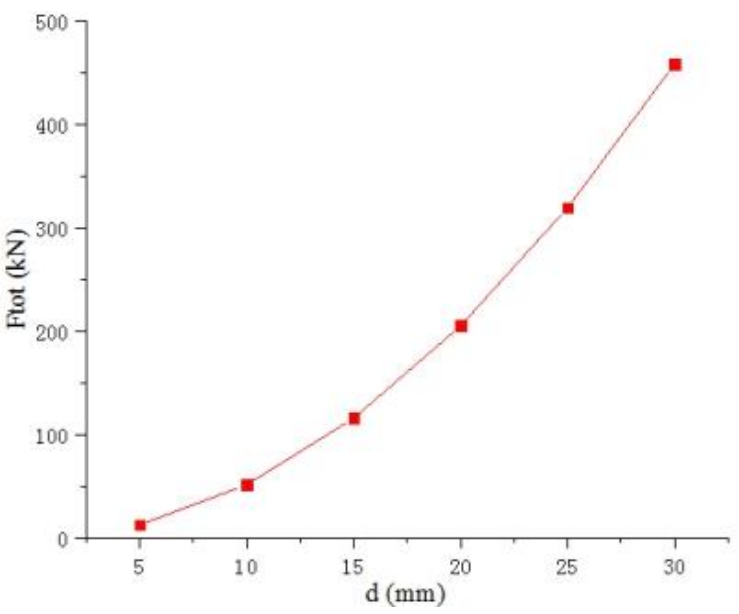

b. Resultant force of different bolt diameter

Fig. 6. Variation of resultant force and resultant displacement of different bolt diameters under $45^{\circ}$ loading angle.

\section{Conclusion}

1. Based on the elastic-plastic mechanics and Winkler foundation beam assumption, the plastic strengthening model is adopted for the bolt and the ideal elastic-plastic model is used for rock. The force-displacement curve of the analysis model from the beginning of loading to the failure of the bolt under a certain displacement loading angle is obtained;

2. Once the bolt is supported in rock with different strength, the deformation and force of ultimate failure decrease gradually with the increase of strength, and have little influence when reaching a certain value;

3. When different bolt diameters are supported in the same rock mass, the displacement at final failure increases linearly with the bolt diameter, and the force increases exponentially with the bolt diameter;

4. According to the calculation model, it can be found that whether the bolt is in failure or about to be damaged can be judged in the actual project, which provides early warning for the support.

\section{Acknowledgments}

Authors wishing to acknowledge research projects supported by the Open Research Fund of Hunan Provincial Key Laboratory of Hydropower Development Key Technology, Grant NO. PKLHD201704 and the Hunan Provincial Natural Science Foundation (Grant NO. 2017JJ2296).

\section{References}

1. F.F. Ren, Z.J. Yang, J.F. Chen, W.W. Chen. An analytical analysis of the full-range behaviour of grouted rockbolts based on a tri-linear bond-slip model. Construction and Building Materials, 24(3): 361-370 (2010)

2. S. Ma, Z. Zhao, W. Nie, et al. An Analytical Model for Fully Grouted Rockbolts with Consideration of the Pre- and Post-yielding Behavior. Rock Mechanics and Rock Engineering, DOI: 10.1007/s00603-017-1272-5 (2017)
3. L. He, X.M. An, X.B. Zhao, et al. Development of a Unified Rock Bolt Model in Discontinuous Deformation Analysis. Rock Mechanics and Rock Engineering, 51(3): 827-847 (2018)

4. F. Pellet, P. Egger. Analytical model for the mechanical behaviour of bolted rock joints subjected to shearing. Rock Mechanics and Rock Engineering, 29(2): 73-97 (1996)

5. A.M. Ferrero. The shear strength of reinforced rock joints. International Journal of Rock Mechanics and Mining Sciences \& Geomechanics Abstracts, 32(6): 595-605 (1995)

6. W. Wang, Q. Song, C. Xu, et al. Mechanical behaviour of fully grouted GFRP rock bolts under the joint action of pre-tension load and blast dynamic load. Tunnelling and Underground Space Technology, 73:82-91 (2018)

7. C.H. Liu, Y.Z. Li. Analytical Study of the Mechanical Behavior of Fully Grouted Bolts in Bedding Rock Slopes. Rock Mechanics and Rock Engineering, 50(9):2413-2423 (2017)

8. C. Carranza-Torres. Analytical and Numerical Study of the Mechanics of Rockbolt Reinforcement around Tunnels in Rock Masses. Rock Mechanics and Rock Engineering, 42(2): 175-228 (2009)

9. Y. Cai, Y. Jiang, I. Djamaluddin, et al. An analytical model considering interaction behavior of grouted rock bolts for convergence-confinement method in tunneling design. Int J Rock Mech Min Sci 76:112126 (2015)

10. C.H. Tan. Difference solution of passive bolts reinforcement around a circular opening in elastoplastic rock mass. Int J Rock Mech Min Sci, 81:28-38 (2016)

11. D. Oliveira, M.S. Diederichs. Tunnel support for stress induced failures in Hawkesbury Sandstone. Tunn Undergr Space Technol, 6, 4:10-23 (2017)

12. Y. Chen. Experimental study and stress analysis of rock bolt anchorage performance. Journal of Rock Mechanics and Geotechnical engineering, 32(5): 428-437 (2014) 